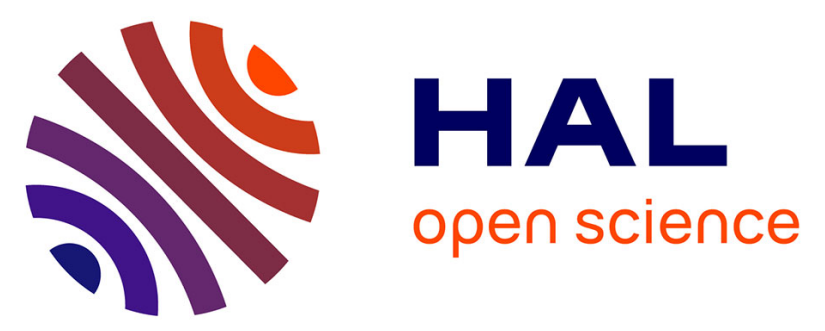

\title{
Nonequilibrium Dynamics Induced by Scattering Forces for Optically Trapped Nanoparticles in Strongly Inertial Regimes
}

\author{
Yacine Amarouchene, Matthieu Mangeat, Benjamin Vidal Montes, Lukas \\ Ondic, Thomas Guérin, David S. Dean, Yann Louyer
}

\section{To cite this version:}

Yacine Amarouchene, Matthieu Mangeat, Benjamin Vidal Montes, Lukas Ondic, Thomas Guérin, et al.. Nonequilibrium Dynamics Induced by Scattering Forces for Optically Trapped Nanoparticles in Strongly Inertial Regimes. Physical Review Letters, 2019, 122 (18), pp.183901 (1-6). 10.1103/PhysRevLett.122.183901 . hal-02125301

\section{HAL Id: hal-02125301 https://hal.science/hal-02125301}

Submitted on 10 May 2019

HAL is a multi-disciplinary open access archive for the deposit and dissemination of scientific research documents, whether they are published or not. The documents may come from teaching and research institutions in France or abroad, or from public or private research centers.
L'archive ouverte pluridisciplinaire $\mathbf{H A L}$, est destinée au dépôt et à la diffusion de documents scientifiques de niveau recherche, publiés ou non, émanant des établissements d'enseignement et de recherche français ou étrangers, des laboratoires publics ou privés. 


\title{
Nonequilibrium Dynamics Induced by Scattering Forces for Optically Trapped Nanoparticles in Strongly Inertial Regimes
}

\author{
Yacine Amarouchene, ${ }^{1, *}$ Matthieu Mangeat, ${ }^{1}$ Benjamin Vidal Montes, ${ }^{1}$ Lukas Ondic, ${ }^{2}$ \\ Thomas Guérin, ${ }^{1}$ David S. Dean, ${ }^{1}$ and Yann Louyer ${ }^{1, \dagger}$ \\ ${ }^{1}$ LOMA, CNRS UMR 5798, University of Bordeaux, F-33400 Talence, France \\ ${ }^{2}$ Institute of Physics, Academy of Sciences of the Czech Republic, CZ-162 00 Prague, Czech Republic
}

(Received 24 September 2018; revised manuscript received 28 March 2019; published 8 May 2019)

\begin{abstract}
The forces acting on optically trapped particles are commonly assumed to be conservative. Nonconservative scattering forces induce toroidal currents in overdamped liquid environments, with negligible effects on position fluctuations. However, their impact in the underdamped regime remains unexplored. Here, we study the effect of nonconservative scattering forces on the underdamped nonlinear dynamics of trapped nanoparticles at various air pressures. These forces induce significant low-frequency position fluctuations along the optical axis and the emergence of toroidal currents in both position and velocity variables. Our experimental and theoretical results provide fundamental insights into the functioning of optical tweezers and a means for investigating nonequilibrium steady states induced by nonconservative forces.
\end{abstract}

DOI: 10.1103/PhysRevLett.122.183901

Classical statistical mechanics establishes that a particle of mass $m$ subject to a potential $V$ in the presence of a heat bath, for instance the surrounding medium such as air or water will, in thermal equilibrium, be described by the Gibbs-Boltzmann probability distribution for its position $\mathbf{x}$ and velocity $\mathbf{v}[1]$

$$
P_{\mathrm{GB}}(\mathbf{x}, \mathbf{v})=\frac{1}{Z_{x v}} \exp \left(-\frac{\frac{1}{2} m \mathbf{v}^{2}+V(\mathbf{x})}{k_{B} T}\right) .
$$

Here, $T$ is the temperature imposed by the heat bath, $k_{B}$ is Boltzmann's constant, and $Z_{x v}$ a normalization constant, better known as the canonical partition function. Equation (1) has some remarkable consequences that are today taken for granted. First, the position $\mathbf{x}$ and the velocity $\mathbf{v}$ are independent random variables. The marginal distribution for the velocity is the celebrated MaxwellBoltzmann distribution, proposed at the very inception of the field of statistical mechanics, and is independent of the interaction potential. Another remarkable property is that the equilibrium distribution is independent of the dynamics. This, today obvious, observation means that at the same temperature and potential, a particle trapped in water will have the same equilibrium distribution as the one trapped in air. Another feature of the Gibbs-Boltzmann distribution is that it does not have any currents in position or velocity; this is necessary in an equilibrium distribution so that it satisfies time reversal symmetry [2].

However, when the force acting on the particle is not derived from a potential, much less is known on the nonequilibrium stationary distribution. Optically trapped particles have recently attracted attention as a model system to study nonequilibrium forces [3-8]. As originally shown by Ashkin [9], a particle can be trapped by using the (conservative) intensity gradient force of a laser. It is also well known that an optically trapped particle is also submitted to nonconservative scattering forces. These forces, first measured in Ref. [8], induce steady-state nonequilibrium probability currents, which develop over time due to thermal fluctuations displacing the particle away from its stable mechanical equilibrium point into the

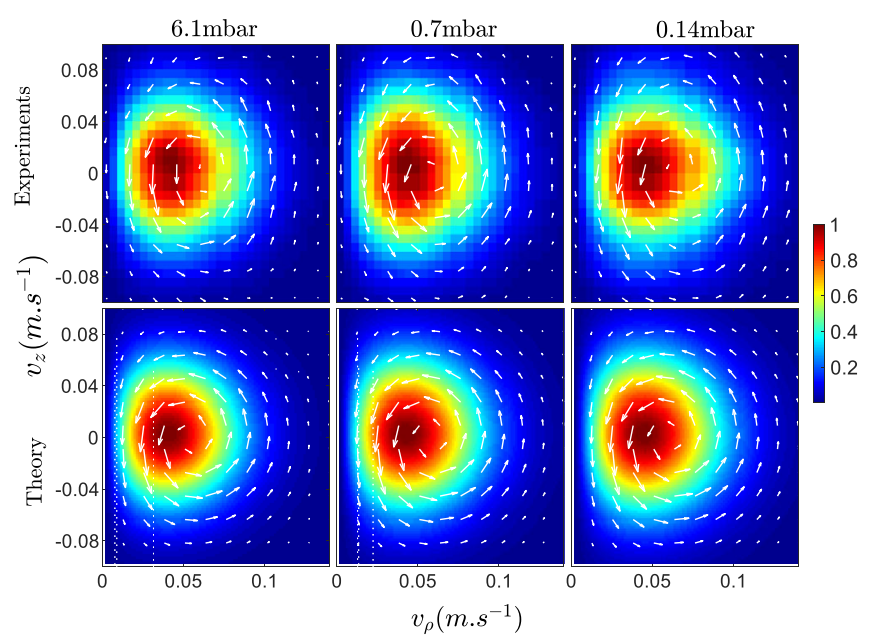

FIG. 1. Experimental and theoretical probability currents $\overline{\mathbf{J}}_{\mathbf{v}}$ in velocity space for several pressures in the (radial $v_{\rho}$, axial $v_{z}$ ) plane. The theory is described in the companion article [20]. The color bar displays the steady-state probability distribution $P_{s}(\mathbf{v})$. 
nonconservative force field. Such currents have been demonstrated only in the overdamped regime [3-5] where the effect of nonconservative forces turns out to be modest $[6,7]$. In the underdamped regime, trapping by intensity gradient forces has recently been used in vacuum [10,11], leading to impressive proposals and experimental results in ultraweak force sensing and fundamental tests of quantum mechanics [12-19]. These studies consider only conservative forces, so that nonequilibrium statistical mechanics of inertial (underdamped) optically trapped particles remains unexplored.

In this Letter, we describe an experimental and theoretical study of an underdamped particle in an optical trap which generates a well-characterized nonconservative force. We show that probability currents exist in both position and velocity space, the latter being inaccessible in the overdamped case. These currents mean that even the Maxwell-Boltzmann distribution is modified by nonconservative forces (see Fig. 1). Furthermore, these modifications are shown to depend on the details of the dynamics, in this case the damping. As well as modification of static quantities, we also show that the resulting steady-state dynamics is drastically modified by the nonconservative force. Namely, we observe, for the first time in an optical trap, pressure-dependent additional low-frequency broadband axial positional fluctuations. Such effects dominate over thermal fluctuations and are well too weak to be observed in the overdamped regime [6,7]. Such lowfrequency contributions in the axial motion originate from the scattering forces and are further amplified by the nonlinear gradient components. This Letter is accompanied by a theoretical article [20].

We first describe our experiment [detailed in the Supplemental Material (SM) [21] that includes Refs. [22,23]], which consists of trapping, in a near vacuum, a $68-\mathrm{nm}$ radius fused silica nanoparticle with a 430-mW tightly focused linearly polarized laser beam at $\lambda=1064 \mathrm{~nm}$ by using a high 0.8 numerical aperture objective. The Gaussian laser beam propagates in the $z$ direction, while $x, y$ are the transverse directions. The pressure can be varied to modify the friction coefficient applied on the bead and thus to explore the effect of inertia on the particle's motion. A new calibration procedure that takes into account the nonlinear aspects of the optical trap is also presented in the SM [21]. This enables us to estimate the geometrical parameters of the trap, the intrinsic damping rate $\Gamma$, but also the size of the trapped particle and center of mass motion temperature $T$. The final trap parameters obtained from this procedure are $w_{x}=0.915 \mu \mathrm{m}$, $w_{y}=1.034 \mu \mathrm{m}$, and $w_{z}=2.966 \mu \mathrm{m}$ (beam waist radii $w_{i=x, y}$ and Rayleigh length $w_{z}$ ). In the following, these parameters are used to compare the theoretical and experimental results.

A crucial advantage of the underdamped system studied here is that experimental resolution allows an accurate and unambiguous determination of both the velocity $\mathbf{V}_{t}$ and the acceleration $\dot{\mathbf{V}}_{t}$, as compared to overdamped systems [3-5]. This enables us to measure the currents both in position and velocity space. By definition, the probability density function $P(\mathbf{x}, \mathbf{v}, t)$ is simply given by the average over stochastic trajectories $P(\mathbf{x}, \mathbf{v}, t)=\left\langle\delta\left(\mathbf{x}-\mathbf{X}_{t}\right) \delta\left(\mathbf{v}-\mathbf{V}_{t}\right)\right\rangle$, with $\mathbf{X}_{t}, \mathbf{V}_{t}$ the instantaneous position and velocity of the particle at time $t$, and $\langle\cdot\rangle$ denoting ensemble averaging. The currents $\mathbf{J}_{\mathbf{X}}$ and $\mathbf{J}_{\mathbf{v}}$ in position and velocity space are defined by considering the general transport equation for this system given by $\partial_{t} P(\mathbf{x}, \mathbf{v}, t)=-\nabla_{\mathbf{x}} \cdot \mathbf{J}_{\mathbf{x}}-\nabla_{\mathbf{v}} \cdot \mathbf{J}_{\mathbf{v}}$. Integrating these currents leads to the definition of effective currents $\overline{\mathbf{J}}_{\mathbf{x}}=\int d \mathbf{v} \mathbf{J}_{\mathbf{x}}=\left\langle\mathbf{V}_{t} \delta\left(\mathbf{x}-\mathbf{X}_{t}\right)\right\rangle$ in position space and $\overline{\mathbf{J}}_{\mathbf{v}}=\int d \mathbf{x} \mathbf{J}_{\mathbf{v}}=\left\langle\dot{\mathbf{V}}_{t} \delta\left(\mathbf{v}-\mathbf{V}_{t}\right)\right\rangle$ in velocity space. These currents are experimentally estimated either by a sixdimensional histogram binning [8] or kernel density techniques [4]. Here, the steady state is reached by using suitably small bin sizes and time averaging.

We consider the marginal probability distribution of the velocity and its associated current, which to our knowledge is measured here for the first time. Shown in Fig. 1 is the steady current in velocity space $\overline{\mathbf{J}}_{\mathbf{v}}$ measured at different pressures (and therefore different friction coefficients). We see that the current (shown as arrows) is nonzero-a clear indication of the presence of a nonconservative force and deviation from the Maxwell-Boltzmann distribution. Interestingly, these currents seem to form rolls (in velocity space) that are in the opposite direction to currents in position space (Fig. 2, inset).

To get a theoretical understanding of these currents, we consider the Langevin dynamics of a trapped particle,

$$
m \ddot{x}_{i}+m \Gamma \dot{x}_{i}=-\kappa_{i} x_{i}+F_{i}^{\text {Duffing }}+F_{i}^{\text {scatt }}+f_{i}(t)
$$

with $m$ the particle mass, $m \Gamma$ the friction coefficient, and $f_{i}(t)$ the thermal white noise force [20]. In addition to the standard harmonic force $-\kappa_{i} x_{i}$ (with $\kappa_{i}$ the stiffness in the direction $i$ ), the trap exerts two additional forces on the particle, which we calculate at next-to-leading order for small displacements relative to the laser wavelength [24]. The term $F^{\text {Duffing }}$ contains the cubic nonlinearities arising from the gradient force and is widely studied in the onedimensional Duffing oscillator $[25,26]$ :

$$
F_{i}^{\text {Duffing }}=\kappa_{i} x_{i}\left[\left(1+\delta_{i, z}\right)\left(\frac{2 x^{2}}{w_{x}^{2}}+\frac{2 y^{2}}{w_{y}^{2}}\right)+\frac{2 z^{2}}{w_{z}^{2}}\right] .
$$

The main contribution of the scattering force is in the axial direction and is given by

$$
F_{i}^{\mathrm{scatt}}=\delta_{i z} \frac{\alpha^{\prime \prime}}{\alpha^{\prime}} \kappa_{z}\left(\gamma_{0}+\sum_{j=x, y, z} \gamma_{j} x_{j}^{2}\right),
$$

where $\alpha^{\prime}$ and $\alpha^{\prime \prime}$ are, respectively, the real and imaginary parts of the effective polarizability $\alpha=\alpha_{0} /\left[1-i k^{3} \alpha_{0} /\left(6 \pi \epsilon_{0}\right)\right]$, 
with $k$ the laser wave vector and $\epsilon_{0}$ the vacuum permittivity [24]. Optical forces arise from the interaction between the electromagnetic field of the Gaussian beam and the polarizability $\alpha_{0}=4 \pi \epsilon_{0} R_{p}^{3}(\epsilon-1) /(\epsilon+2)$ of a spherical object of dielectric constant $\epsilon$ and radius $R_{p}$. The coefficients $\gamma_{i}$ depend on the geometrical parameters of the focused laser: $\gamma_{0}=w_{z}\left(w_{z} k-1\right), \gamma_{i=x, y}=k / 2-2 \gamma_{0} / w_{i}^{2}$, and $\gamma_{z}=$ $\left(2-w_{z} k\right) / w_{z}[24]$.

Importantly, these scattering forces are nonconservative. In the overdamped regime, these forces are known to give rise to nonequilibrium probability currents that have been termed Brownian vortices, and arise in a minimal scattering model (MSM), which assumes an axisymmetric trap (i.e., $\gamma_{x}=\gamma_{y}$ and $\kappa_{x}=\kappa_{y}=\kappa_{\perp}$ ), and disregards Duffing nonlinearities as well as the axial term $\left(\gamma_{z} z^{2}\right)$ in the scattering force. In the MSM, this force can then be written as

$$
F_{i}^{\text {scatt }}=\delta_{i z} \varepsilon \kappa_{\perp} a\left[1-\left(x^{2}+y^{2}\right) / a^{2}\right],
$$

where the dimensionless parameter $\varepsilon$, which quantifies the magnitude of scattering forces, and the length scale $a \simeq w_{\perp} / \sqrt{2}$ at which scattering forces vary, are easily identified by comparing with (4).

Until now, studies of this MSM model considered overdamped motion [6]; here we extend the study to inertial particles. The theory to perform this task is described in the companion paper [20]. In this model the fluxes can be exactly computed at first order in $\varepsilon$,

$\overline{\mathbf{J}_{\mathbf{x}}}=\varepsilon A_{x}\left[\frac{\kappa_{z}}{\kappa_{\perp}} z \rho \hat{\mathbf{e}}_{\rho}+\left(\frac{2 k_{B} T}{\kappa_{\perp}}-\rho^{2}\right) \hat{\mathbf{e}}_{z}\right] e^{-\left(\kappa_{\perp} \rho^{2}+\kappa_{z} z^{2}\right) / 2 k_{B} T}$,

$\overline{\mathbf{J}}_{\mathbf{v}}=-\varepsilon A_{v}\left[v_{z} \mathbf{v}_{\rho}+\left(\frac{2 k_{B} T}{m}-\mathbf{v}_{\rho}^{2}\right) \hat{\mathbf{e}}_{z}\right] e^{-m \mathbf{v}^{2} / 2 k_{B} T}$

where the analytical expressions for the (positive) amplitude factors $A_{x}$ and $A_{v}$ are explicitly given in [20], with $\rho$ the distance to the optical axis and $\mathbf{v}_{\rho}$ the transverse component of the velocity. The geometry of fluxes in position space is the same as for overdamped systems, but interestingly the amplitude factor is found to be nonmonotonic with the friction coefficient. A similar geometry, but with opposite sign, holds in velocity space.

In both position and velocity spaces, the predictions for the current geometry are very close to the experimental observations shown in Figs. 1 and 2 (inset). The comparison can be made quantitative by defining a scalar quantity characterizing the amplitude of the fluxes. We thus define $\left\langle{\overline{\mathbf{J}_{\mathbf{v}}}}^{2}\right\rangle$ as the uniform average of the squared flux over the window of velocities shown in Fig. 1. We represent in Fig. 2 the pressure dependence of the amplitude $\left\langle{\overline{\mathbf{J}_{\mathbf{v}}}}^{2}\right\rangle^{1 / 2}$ and compare with the MSM theory. As predicted by the theory [20], the current's amplitude saturates at low

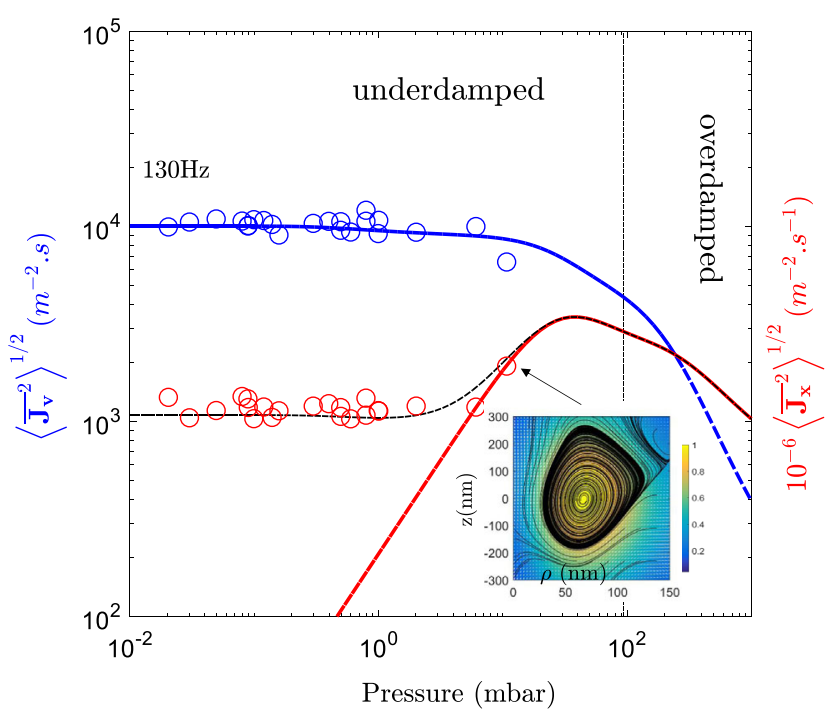

FIG. 2. In blue, experimental (circle) and theoretical minimal scattering model MSM (line, [20]) probability current amplitudes $\left\langle\overline{\mathbf{J}}_{\mathbf{v}}{ }^{2}\right\rangle^{1 / 2}$ in velocity space for several pressures, obtained by a uniform average over the velocity window shown in Fig. 1. Similarly, experimental and theoretical MSM current amplitudes in the position space are shown in red. The theory uses parameters obtained from the calibration [21]. The dashed line is obtained from Eq. (6) via an effective damping constant as seen in [21]. Also shown as an inset is the current map in position space measured at 10.8 mbar.

pressure at a value that corresponds to the theory if one uses the parameter values arising from the calibration technique. We find $\varepsilon=0.05$ consistent with the value of the imaginary index of fused silica. The corresponding circulation rate for these vortices in velocity space is about $\Omega_{0}=(1 / 2 \pi) \int d \mathbf{v}\left(\boldsymbol{\nabla}_{\mathbf{v}} \times \mathbf{J}_{\mathbf{v}}\right) \cdot \mathbf{e}_{\theta} \approx 130 \mathrm{~Hz}$. The quantitative success of the MSM theory, which neglects Duffing nonlinearities, is likely due to the fact that the marginal distribution is dominated by positions close to the trap center where nonlinear effects are by definition small. In position space, rather than following the MSM theoretical prediction, we see that the amplitude $\left\langle{\overline{\mathbf{J}_{\mathbf{x}}}}^{2}\right\rangle^{1 / 2}$ saturates at low pressure (Fig. 2). This shows that Duffing nonlinearities, absent in the MSM theory, are important to quantitatively describe fluxes in position space. Such nonlinearities lead to a saturation of effective damping rates seen in the spectral densities at the oscillator eigenfrequencies [21]. Interestingly, using this effective damping rate $\Gamma_{\text {eff }}$ [identified in Fig. S2(e) of [21] ] leads to a good agreement with the data shown in Fig. 2. This, however, deserves further experimental and theoretical investigation.

In what follows, we present evidence that scattering forces make a dominant contribution to a dynamic quantity: the power spectral density (PSD) $S_{z z}(\omega)$ in the longitudinal direction. Within the MSM, $S_{z z}(\omega)$ displays a lowfrequency peak and is exactly given for low pulsations $\omega$ by $[20]$ : 


$$
S_{z z}(\omega)=\frac{2 k_{B} T \Gamma}{m \Omega_{z}^{4}}+4\left(\frac{\varepsilon k_{B} T}{a m \Omega_{z}^{2}}\right)^{2} \frac{\Gamma}{\Gamma^{2}+\omega^{2}},
$$

where $\Omega_{z}=\left(\kappa_{z} / m\right)^{1 / 2}$. The first term in the expression of $S_{z z}(\omega)$ corresponds to the thermal component and the second term is due to nonconservative forces. Its origin stems from the fact that $x^{2}(t)$ and $y^{2}(t)$ contain weakly varying functions, giving rise to a Lorentzian low-frequency component. This is similar to the analysis of Ref. [25]. If we compare the two terms, the above equation implies that the low frequency part of the spectrum becomes increasingly dominated by the component due to scattering forces in the underdamped limit. This is a major difference with the overdamped case, where the effect of these scattering forces are insignificant at all frequencies [6].

However, Duffing nonlinearities are neglected in the MSM, which can appear as naive since these nonlinearities are well known to be essential to describe one-dimensional underdamped oscillators [24]. To determine whether the above predictions hold in a more realistic model, we now turn to Langevin simulations of the 3D fully nonlinear Eq. (2) and calculate the respective PSDs [27]. These simulations are performed for parameter values obtained by the trap calibration [21]. Here, we distinguish between different situations: the Duffing case, where we neglect the scattering force; the fully nonlinear model (FNL) includes all terms of Eq. (2), and the analytically soluble MSM which neglects Duffing nonlinearities. We note that our numerical simulations reveal that the transverse scattering forces $F_{i=x, y}^{\text {scatt }}$ have a negligible contribution in the frequency and position fluctuations, which is why we omit them in Eq. (4).

Figures 3(a) and 3(b) show PSDs in the axial and transverse directions. We see how the scattering force increases the frequency shift of the resonance by a few percent. More importantly, the low-frequency peak is present in the MSM (as expected) but completely absent in the Duffing model, which shows that nonconservative forces are necessary for its emergence. In the complete nonlinear model, this peak is not only still present, but is also largely amplified (when compared to the MSM). Note that these low-frequency overdamped fluctuations are absent in the transverse spectral densities (data not shown). For comparison with previous works in analog circuits [28], we also represent a tilted Duffing case, in which case Duffing terms and the linear part of the scattering force in Eq. (4) are used. This tilted Duffing model clearly overestimates the low-frequency response [Fig. 3(a)]. Equation (8) perfectly reproduces the numerical simulation of $S_{z z}(\omega)$ for the MSM [see Fig. 3(a) and [20] ] and shows that the low-frequency overdamped component (only visible in the axial direction) has a corner frequency given by $\Gamma$. The $\Gamma$ dependence of the amplitude $S_{z z}(0)$ also holds for the different cases described above, as seen in
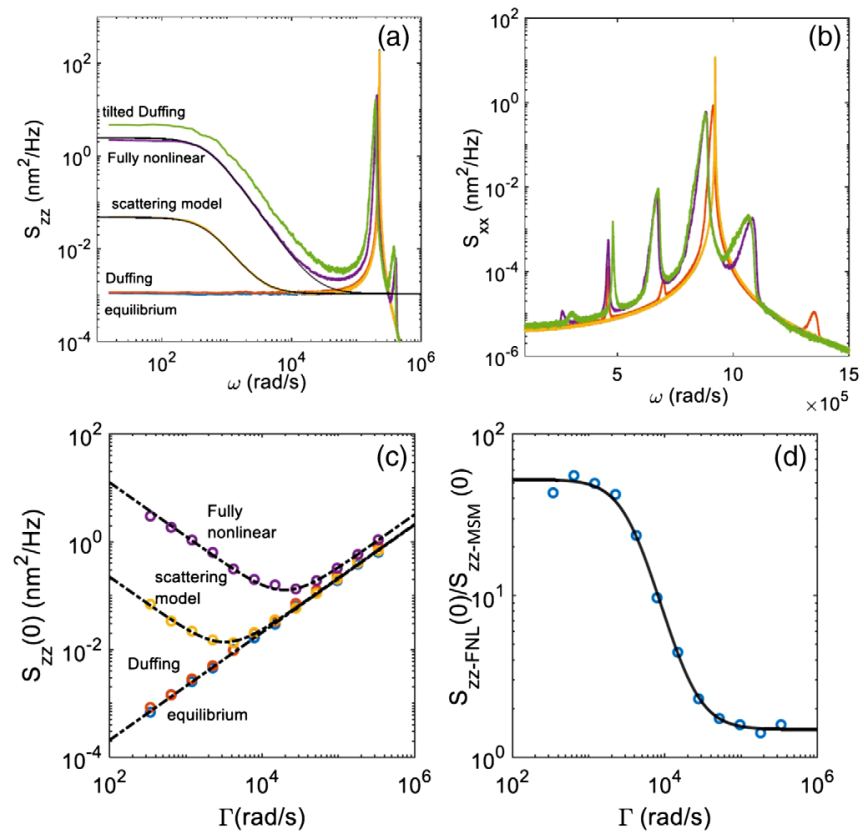

FIG. 3. Numerical simulation results using the waists $w_{i}$ extracted from the nonlinear calibrations at a pressure of $0.1 \mathrm{mbar}$ (i.e., for $\Gamma=500 \mathrm{rad} / \mathrm{s}$ ). PSDs $S_{i i}$ along $i=z$ axis (a) and $i=x$ axis (b) for the different cases described in the text [the same color legend shown in Fig. 3(a) is used for all the figures]. (c) $S_{z z}(0)$ versus damping rate $\Gamma$. (d) Ratio of the zero frequency response $S_{z z}(0)$ for the fully nonlinear model and the MSM (see text).

Fig. 3(c). Strikingly, the theory also gives good fitting results for the fully nonlinear model at low frequency provided that a pressure-dependent correction factor $S_{z z-\mathrm{FNL}}(0) / S_{z z-\mathrm{MSM}}(0)$ [shown in Fig. 3(d)] is used solely for the scattering term in Eq. (8).

Finally, we investigate experimentally the presence of a low-frequency component in the axial PSD due to scattering forces. In practice, the low-frequency $1 / f$-like noise of the laser hinders the direct observation of $S_{z z}(0)$. To circumvent this technical issue, Fig. 4(a) displays the raw experimental PSDs $S_{i i}(\omega)=c_{x} S_{x x}(\omega)+c_{z} S_{z z}(\omega)$ for a limited range of frequencies at $0.8 \mathrm{mbar}\left(c_{x}\right.$ and $c_{z}$ being calibration constants). These experimental data are in good agreement with our numerical simulations using the calibration factors and the beam parameters [21]. Figure 4(b) displays the pressure dependence of the lowfrequency response of the PSDs along the $z$ axis. Knowing $\Gamma$ for each pressure, the fitting of Eq. (8) enables us to infer $S_{z z}(0)$ relative to its equilibrium value $S_{z z-\mathrm{eq}}(0)$ as shown in Fig. 4(c). The scaling $S_{z z}(0) / S_{z z-\mathrm{eq}}(0) \propto \Gamma^{-2}$, which is expected from the MSM, is also observed at low pressures. Using corrections in temperature shown in Fig. S2(d) of [21] and the correction factor of Fig. 3(d) provides a means to estimate $\varepsilon \approx 0.04$ corresponding to an imaginary refractive index of $\approx 10^{-7}$, as expected for fused silica [29] and in 

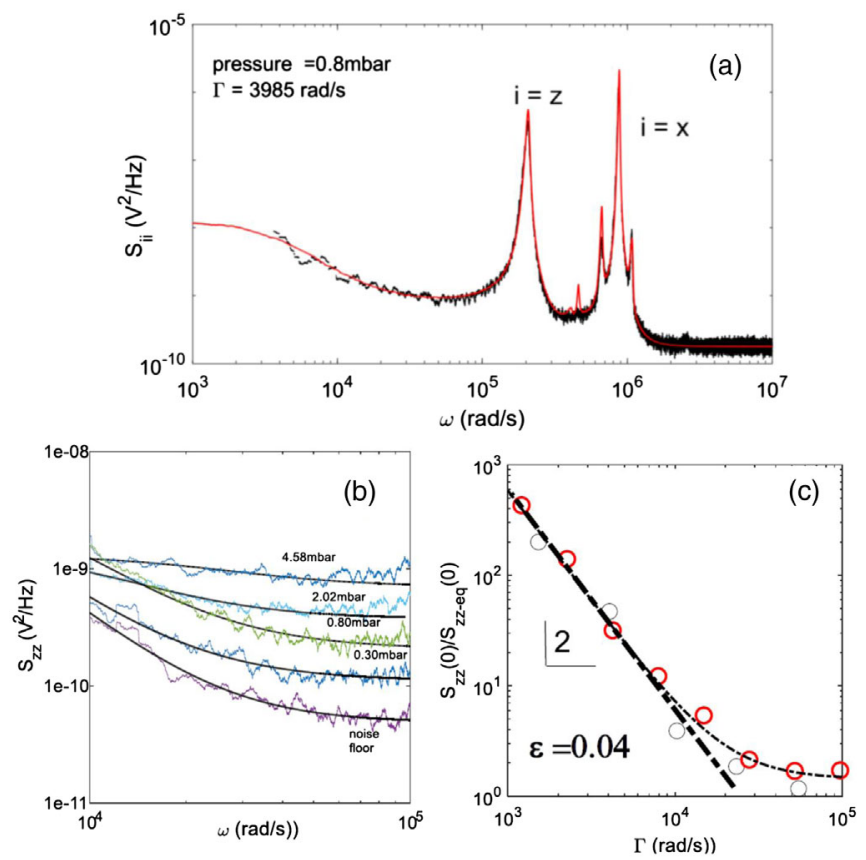

FIG. 4. (a) Experimental PSDs (in directions $x$ and $z$ ) versus fully nonlinear simulation of the PDSs at a pressure of 0.8 mbar [black experiments and red simulations, idem for (c)]. (b) Lowfrequency part of $S_{z z}(\omega)$ at various pressures. The fits to the data correspond to a fit using the functional form of Eq. (8). The deduced values of $S_{z z}(0)$ relative to the equilibrium value versus the damping rate $\Gamma$ are shown in (c). The thin line is a guide to the eye while the thick line highlights the $\Gamma^{2}$ scaling discussed in the text.

agreement with the previous value of $\varepsilon$ used to describe the fluxes amplitude.

In summary, we have experimentally and theoretically demonstrated the effect of radiation pressure for optically trapped nanoparticles in the nonlinear underdamped regime. In a near vacuum environment, position fluctuations are amplified at low pressure. Toroidal Brownian vortices in both position and velocity space have been observed. The currents in position space, however, deserve further theoretical and experimental study. In particular, understanding the exact topology of Brownian vortices (in position-velocity space) and their efficiencies versus dissipation requires further experimental investigation in the underdamped regime [5], but also paves the way towards studying the time reversal symmetry breaking induced by nonconservative forces [20]. Our work opens a new pathway for studying nonequilibrium statistical physics for a wide range of damping regimes. It also highlights the importance of fully characterizing optical traps in the underdamped regime that is relevant for the quest of ultraweak force sensing and fundamental tests of quantum mechanics.

We thank L. Haelman for the construction of the vacuum chamber, W. Benharbone, S. Cassagnere, and B. Tregon from the Electronics Lab, and R. Avriller for stimulating discussions. This work was partially funded by the Bordeaux IdEX program-LAPHIA (ANR-10-IDEX-0302)-Arts et Science (Sonotact 2017-2018) and Projet Region Aquitaine (2018-1R50304).

*yacine.amarouchene@u-bordeaux.fr yann.louyer@u-bordeaux.fr

[1] D. A. McQuarrie, Statistical Mechanics (Harper and Row, New York, 1975).

[2] D. Ruelle, Thermodynamic Formalism: The Mathematical Structures of Classical Equilibrium Statistical Mechanics (Cambridge University Press, Cambridge, England, 2004).

[3] Y. Roichman, B. Sun, A. Stolarski, and D. G. Grier, Phys. Rev. Lett. 101, 128301 (2008).

[4] B. Sun, J. Lin, E. Darby, A. Y. Grosberg, and D. G. Grier, Phys. Rev. E 80, 010401(R) (2009).

[5] H. W. Moyses, R. O. Bauer, A. Y. Grosberg, and D. G. Grier, Phys. Rev. E 91, 062144 (2015).

[6] M. de Messieres, N. A. Denesyuk, and A. La Porta, Phys. Rev. E 84, 031108 (2011).

[7] G. Pesce, G. Volpe, A. C. De Luca, G. Rusciano, and G. Volpe, Europhys. Lett. 86, 38002 (2009).

[8] P. Wu, R. Huang, C. Tischer, A. Jonas, and E.-L. Florin, Phys. Rev. Lett. 103, 108101 (2009).

[9] A. Ashkin, Optical Trapping and Manipulation of Neutral Particles Using Lasers (World Scientific Publishing, Singapore, 2006), and references therein.

[10] T. Li, S. Kheifets, and M. G. Raizen, Nat. Phys. 7, 527 (2011).

[11] J. Gieseler, B. Deutsch, R. Quidant, and L. Novotny, Phys. Rev. Lett. 109, 103603 (2012).

[12] A. Arvanitaki and A. A. Geraci, Phys. Rev. Lett. 110, 071105 (2013).

[13] A. Bassi, K. Lochan, S. Satin, T. Singh, and H. Ulbricht, Rev. Mod. Phys. 85, 471 (2013).

[14] J. Bateman, S. Nimmrichter, K. Hornberger, and $H$. Ulbricht, Nat. Commun. 5, 4788 (2014).

[15] D. C. Moore, A. Rider, and G. Gratta, Phys. Rev. Lett. 113, 251801 (2014).

[16] G. Ranjit, D. P. Atherton, J. H. Stutz, M. Cunningham, and A. A. Geraci, Phys. Rev. A 91, 051805(R) (2015).

[17] A. D. Rider, D. C. Moore, C. P. Blakemore, M. Louis, M. Lu, and G. Gratta, Phys. Rev. Lett. 117, 101101 (2016).

[18] G. Ranjit, M. Cunningham, K. Casey, and A. A. Geraci, Phys. Rev. A 91, 051805 (2015); 93, 053801 (2016).

[19] J. Liu and K.-D. Zhu, Phys. Rev. D 95, 044014 (2017).

[20] M. Mangeat, Y. Amarouchene, Y. Louyer, T. Guérin, and D. S. Dean, Phys. Rev. E 99, 052107 (2019).

[21] See Supplemental Material at http://link.aps.org/ supplemental/10.1103/PhysRevLett.122.183901 for full details in the experimental setup and calibration method.

[22] S. A. Beresnev, V. G. Chernyak, and G. A. Fomyagin, J. Fluid Mech. 219, 405 (1990).

[23] A. Rohrbach, Phys. Rev. Lett. 95, 168102 (2005).

[24] J. Gieseler, L. Novotny, and R. Quidant, Nat. Phys. 9, 806 (2013); M. Yoneda and K. Aikawa, J. Phys. B 50, 245501 (2017). 
[25] M. I. Dykman and M. A. Krivoglaz, Phys. Status Solidi B 48, 497 (1971); Physica (Amsterdam) 104A, 495 (1980). [26] W. Renz, Z. Phys. B 59, 91 (1985).

[27] D. A. Sivak, J. D. Chodera, and G. E. Crook, J. Phys. Chem. B, 118, 6466 (2014).
[28] M. I. Dykman, R. Mannella, P. V. E. McClintock, S. M. Soskin, and N. G. Stocks, Phys. Rev. A 43, 1701 (1991); 42, 7041 (1990).

[29] R. Kitamura, L. Pilon, and M. Jonasz, Appl. Opt. 46, 8118 (2007). 